\title{
A rare case, diagnosed as calcified callosal lipoma, when the patient presented with acute stroke
}

Zeynep Özözen Ayas ${ }^{1}$, Dilcan Kotan², Pınar Polat ${ }^{3}$

${ }^{1}$ Department of Neurology, Sakarya University Training and Research Hospital, Sakarya, Turkey

${ }^{2}$ Department of Neurology, Medical Faculty, Sakarya University, Sakarya, Turkey

${ }^{3}$ Department of Radiology, Koşuyolu Medipol Hospital, İstanbul, Turkey

Submitted: 24 March 2016

Accepted: 16 April 2016

Arch Med Sci Artheroslcer Dis 2016; 1: e6-e8

DOI: 10.5114/amsad.2016.59586

Copyright @ 2016 Termedia \& Banach

Intracranial lipomas are rare and generally located at pericallosal midline lesions. They are usually asymptomatic; when symptoms occur, they may depend on localization and include headache, seizure, psychomotor retardation, and cranial nerve deficits [1]. These mass lesions, which can be diagnosed with computed tomography (CT) or magnetic resonance imaging (MRI), cannot be treated with radical surgery, because they are usually adherent to the surrounding neurovascular structures and asymptomatic, and surgical excision may result in high mortality and morbidity risk. Incidental asymptomatic lipomas are not treated with surgical excision. However, surgery should be considered in the case of uncontrollable seizures, hydrocephalus, progressive dementia and increased intracranial pressure. In this article, we present a rare case of incidental pericallosal lipoma detected by neuroimaging at the time of consulting for acute cerebrovascular disease.

A 47-year-old man presented to the emergency department with paresthesia in the right arm and leg for a day. His past medical history was notable for hypertension with a regular use of antihypertensive medications. His family history was not remarkable for any disorder. Neurological examination revealed minimal hemiparesia and hemihypoesthesia on the right side. Routine laboratory tests and electrocardiography were normal. Cranial CT revealed agenesis of the corpus and splenium of the corpus callosum as well as a lipoma located in the corpus callosum (Figure 1 A). Diffusion MRI and apparent coefficient (ADC) MRI and B100 sections also showed a subacute hemorrhagic infarction at the level of the left centrum semiovale, which extended to the external capsule (Figures $1 \mathrm{~B}-\mathrm{C})$. Contrast-enhanced brain MRI showed a non-contrast enhanced mass lesion consistent with lipoma that appeared hyperintense on T1 and FLAIR sequences and iso-hyperintense on T2 sequences (Figures $1 \mathrm{D}-\mathrm{F})$. Imaging protocols performed for acute stroke and incidentally detected a pericallosal lipoma, which is a rare tumor. Echocardiography and carotid vertebral Doppler ultrasound examination performed for determination of the etiology of stroke were not remarkable for any pathology. He was asymptomatic, so surgical excision was not considered, and he was treated with oral antiaggregant therapy for stroke.

Intracranial lipoma is a rare tumor, with a frequency of less than $0.1 \%$ among all intracranial tumors [2]. It usually derives from the pericallosal cistern and is designated as a corpus callosum lipoma. It is located in

\author{
Corresponding author: \\ Dilcan Kotan \\ Department of Neurology \\ Medical Faculty \\ Sakarya University \\ 25050 Sakarya, Turkey \\ Phone: 4882143030 \\ E-mail: dilcankotan@ \\ yahoo.com
}



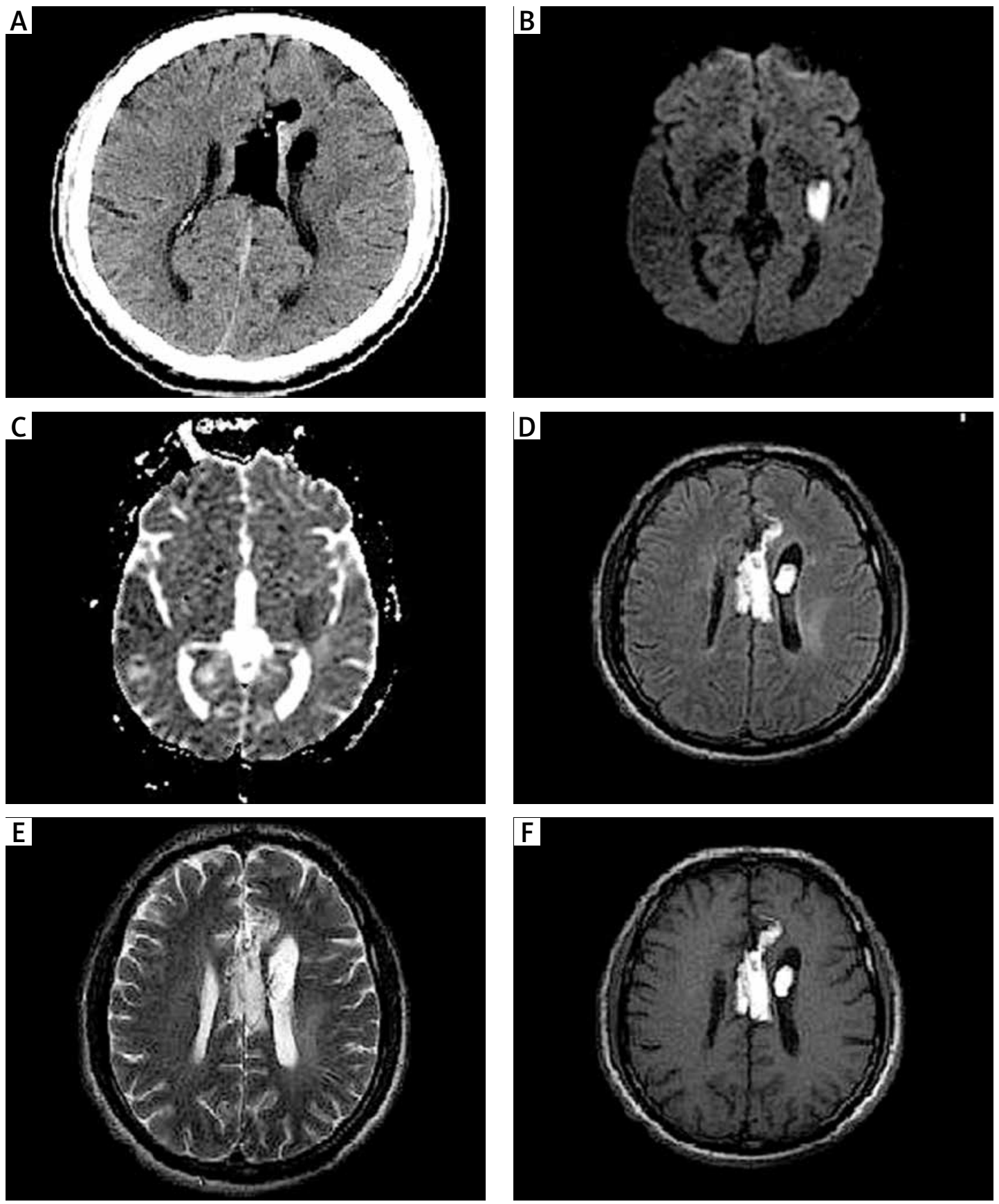

Figure 1. A - Cranial CT revealed agenesis of the corpus and splenium of corpus callosum as well as a lipoma located in corpus allosum as well as a lipoma located in corpus callosum. B, C - Diffusion MRI and apparent coefficient (ADC) MRI and B100 sections also showed a subacute hemorrhagic infarction at the level of left centrum semiovale, which extended to capsula externa. D-F - Contrast enhanced MRI showed a non-contrast enhanced mass lesion consistent with lipoma that appeared hyperintense on T1 and FLAIR sequences and iso-hyperintense on $\mathrm{T} 2$ sequences

the interpeduncular, sylvian, superior cerebellar, quadrigeminal, and chiasmatic cistern; the cerebellopontine angle; and the internal auditory meatus or convexity. Despite having an unclear etiopathogenesis, it is considered as a congenital developmental malformed structure. One theory concerning its origin states that embryological primitive meninges originating from neural crest mesenchyma are converted into fatty tissue and differentiate into lipoma [3]. Since it originates from the meninges, intracranial lipoma is almost always located in the subarachnoid space. Wrongly differentiated subarachnoid space (lipoma) may cause cortical dysplasia by preventing the development of cortical tissue [4]. This embryogenic view of lesion pathogenesis explains the frequent association between this lesion and callosal and other brain hypoplasias. Intracranial 
lipomas are frequently accompanied by various central nerve system anomalies [2, 4]. The most frequent one among these is corpus callosum agenesis, while other anomalies include absent septum pellucidum, cranium bifidum, spina bifi$\mathrm{da}$, encephalocele, myelomeningocele, hypoplastic vermis, and cortical malformations [4]. Our case also had corpus callosum agenesis accompanying callosal lipoma.

Intracranial lipoma is usually asymptomatic [1, 3]. Depending on its localization, cerebellopontine angle lipoma is the most symptomatic one, with a symptom frequency of $80 \%$. In addition, callosal and sylvian fissure lipomas are symptomatic in $50 \%$ of cases, and quadrigeminal and ambient cistern lipomas in $20 \%$ of cases [1]. Infundibular and chiasmatic lipomas are frequently asymptomatic [1]. Symptomatic cases may occur with headache, dizziness, seizure, ataxia, visual disturbance, diplopia, impaired hearing, or hearing loss [5]. Güneyli et al. reported that only 16 of their 57 cases were symptomatic, of whom 9 patients had headache [6]. Our patient was asymptomatic and he was incidentally detected to have intracranial lipoma in imaging examinations performed for acute stroke.

Initially diagnosed by autopsy series, intracranial lipomas have become more prevalent due to advances in CT and MR imaging. Lipomas appear as homogeneous, sharply circumscribed, hypodense, and non-contrast enhanced masses on CT [2]. On MRI, they appear hyperintense on T1 and FLAIR sequences, and hypo-isointense on $\mathrm{T} 2$ sequences, and they show no contrast enhancement [3]. Fat suppression technique may be applied for a detailed diagnosis, where they show signal loss. Intracranial lipomas may be confused with dermoid cysts, teratomas, and subacute hematoma on MRI $[2,7,8]$. While dermoid cysts and teratomas frequently have a heterogeneous signal density, lipomas appear homogeneous [1, 2]. On T2-weighted images of fast spin echo (FSE) lipomas appear hyperintense or hypointense, whereas they appear hyperintense on FLAIR images. Hyperintense appearance of lipomas on FSE T2-weighted and FLAIR sections may be confused with subacute hematoma. Our patient was diagnosed with a non-contrast enhanced pericallosal lipoma that appeared hyperintense on T1 and FLAIR sequences and iso-hyperintense on T2 sequences of MRI.

In interhemispheric lipomas, particularly within the fibrous capsule covering the lipoma, calcifications are common [9]. These calcifications may appear as curvilinear lines, in a nodular pattern in the periphery or center of a lipoma. Güneyli et al. detected calcification in only 3 of 57 cases [6]. We observed linear calcifications in the periphery of the patient's lipoma.

The surgical treatment of lipoma is extremely difficult because they are adherent to surrounding tissues and they surround vessels and nerves [5, 7]. Surgical treatment should only be considered in the case of symptomatic progression, growth of mass, increased intracranial pressure, or hydrocephalus. We considered no surgical treatment for our patient since he was asymptomatic and had no compressive symptoms.

Intracranial lipoma is a rare and usually asymptomatic lesion. Its diagnosis has become easier as a result of wider utilization of $C T$ and MRI in neurology practice. It is important to diagnose these masses, which have mostly benign clinical features and may be encountered in clinical practice, and evaluate them, as their characteristics must be well known.

In conclusion, this rare presentation of calcified pericallosal lipoma emphasized that is necessary to diagnose and differentiate acute stroke.

\section{Conflict of interest}

The authors declare no conflict of interest.

\section{References}

1. Maiuri F, Cirillo S, Simonetti L, De Simone MR, Gangemi M. Intracranial lipomas: diagnostic and therapeutic considerations. J Neurosurg Sci 1988; 32: 161-7.

2. Kazner E, Stochdorph O, Wende S, Grumme T. Intracranial lipoma: diagnostic and therapeutic considerations. J Neurosurg 1980; 52: 234-45.

3. Truwit CL, Barkovich AJ. Pathogenesis of intracranial lipoma. An MR study in 42 patients. AJNR 1990; 11: 665-74.

4. Sasaki H, Yoshida K, Wakamoto H, Otani M, Hori C, Toya S. Lipomas of the frontal lobe. Clin Neurol Neurosurg 1996; 98: 27-31.

5. Tankere F, Vitte E, Dunverneuil NM, Soudant J. Cerebellopontine angle lipomas: report of four cases and review of the literature. Neurosurgery 2002; 50: 626-32.

6. Güneyli S, Keleşoğlu KS, Sivri M, Erdoğan H, Koplay M. Intracranial lipomas: lesion localizations, computed tomography and magnetic resonance imaging findings. Genel Tıp Derg 2015; 25: 126-31.

7. Feldman RP, Marcovici A, Lasala PA. Intracranial lipoma of the syvian fissure. J Neurosurg 2001; 94: 515-9.

8. Bakshi R, Shaikh ZA, Kamran S, Kinkel PR. MRI Finding in 32 consecutive lipomas using conventional and advanced sequences. J Neuroimaging 1999; 9: 134-40.

9. Barkovich AJ. Congenital malformations of the brain and skull. In: Pediatric neuroimaging. Barkovich AJ (ed.). 3rd edn. Lippincott Williams \& Wilkins, Philadelphia 2000; 265-66. 